\title{
Permutation Betting Markets: Singleton Betting with Extra Information
}

\author{
Mohammad Ghodsi \\ Sharif University of \\ Technology \\ ghodsi@sharif.edu
}

\author{
Hamid Mahini \\ Sharif University of \\ Technology \\ mahini@ce.sharif.edu \\ Morteza \\ Zadimoghaddam \\ Sharif University of \\ Technology \\ zadimoghaddam@ce.sharif.edu
}

\author{
Vahab S. Mirrokni \\ Microsoft Research \\ One Microsoft Way \\ Redmond, WA 98052 \\ mirrokni@theory.csail.mit.edu
}

\begin{abstract}
We study permutation betting markets, introduced by Chen, Fortnow, Nikolova, and Pennock [3]. For these markets, we consider subset bettings in which each trader can bet on a subset of candidates ending up in a subset of positions. We consider the revenue maximization problem for the auctioneer in two main frameworks: the risk-free revenue maximization (studied in [3]), and the probabilistic revenue maximization. We also explore the use of some certain knowledge or extra information about the possible outcomes of the market. We first show that finding the optimal revenue in the risk-free model for the subset betting problem is inapproximable. This resolves an open question posed by Chen et al. [3]. In order to identify solvable variants of the problem, we propose the singleton betting language which allows traders to bet an arbitrary value on one candidate for one position. For singleton bettings, we first provide a lineartime implementable necessary and sufficient condition for existence of a solution with positive revenue for any possible outcome. Furthermore, we develop an LP-based polynomialtime algorithm to find the optimum solution of this problem. In addition, we show how to extend this LP-based method to handle some extra information about the possible outcomes. Finally, we consider the revenue maximization problem in a probabilistic setting. For this variant, we observe that the problem of maximizing the expected revenue is polynomialtime solvable, but we show that maximizing the probability of achieving a pre-specified revenue is \#P-Complete.
\end{abstract}

Permission to make digital or hard copies of all or part of this work for personal or classroom use is granted without fee provided that copies are not made or distributed for profit or commercial advantage and that copies bear this notice and the full citation on the first page. To copy otherwise, to republish, to post on servers or to redistribute to lists, requires prior specific permission and/or a fee.

EC'08, July 8-12, 2008, Chicago, Illinois, USA.

Copyright 2008 ACM 978-1-60558-169-9/08/07 ...\$5.00.

\section{Categories and Subject Descriptors}

F.2 [Theory of Computation]: Analysis of Algorithms and Problem Complexity; J.4 [Computer Applications]: Social and Behavioral Sciences-Economics

\section{General Terms}

Algorithm, Theory, Economics

\section{Keywords}

Prediction Markets, Revenue Maximization, Betting Markets, Linear Programming, Combinatorial Algorithms, Matching Markets

\section{INTRODUCTION}

Aggregating users' prediction of the outcome of a market has been proved very useful in predicting the future. An effective way of extracting users' prediction of the market is observing users' investment on securities. Investment on financial securities such as investment in stock markets is one of the examples of such phenomena. These investments are analogous to betting in a financial security. Recently, betting markets have been investigated as a tool to effectively collect the wisdom of the crowd in the market. It has been observed that predictions based on such betting markets are more accurate than other forecasts based on other alternatives such as voting $[19,20,1,6,17]$. The key reason behind such increase in accuracy is that betting markets incentivize traders to investigate various aspects of the future events, and make precise decisions.

A central problem in betting markets is the problem of matching traders' bets without incurring risk to the auctioneer. The goal in this problem is to find a set of bets such that for any outcome, there is a surplus in the investment of the traders. This problem can be formalized as the revenue maximization problem for the auctioneer in the risk-free setting. Moreover, matching traders' orders as described above helps in understanding their use in prediction markets. A set of bets that incurs a risk-free positive revenue for the auctioneer indicates cyclic structures or contradictory bets in these markets. On the other hand, non-existence of such set of bets that guarantee a positive revenue for the auctioneer 
may indicate that the users have similar opinions about the outcome of the market, thus it may result in more accurate prediction of the outcome. This analogy shows a relation between the revenue maximization problem for the auctioneer and the quality of the market prediction based on a set of bets. Therefore, the revenue maximization problem in these settings could have applications in evaluating the accuracy of the such predictions.

In this paper, we study the revenue maximization problem in special betting markets known as permutation markets, first studied by Chen et. al [3]. In these markets, the outcome is a permutation of a set of candidates $V$. Traders invest or bet on various securities. The auctioneer collects these bets and either accepts or rejects them. The goal of the auctioneer is to find a subset of bets that incurs a positive surplus, or to find a subset of bets that maximizes his positive surplus, a.k.a. the revenue.

\subsection{Our Contribution}

We study the following two main frameworks in the context of permutation betting markets:

1. The risk-free setting: This framework is defined based on the framework of Chen et.al [3], and the idea of robust optimization under uncertainty [5, 15, 8].

2. The probabilistic setting: This framework is defined based on the idea of stochastic multi-stage optimization $[13,10,23]$.

In the risk-free setting, the goal is to find a subset of traders' bets that guarantees a maximum revenue for the auctioneer in any outcome. This follows the exact setting studied by Chen et. al [3]. This framework is similar to the robust optimization in which the goal is to find a strategy that tends to maximize the objective function in the worst scenario $[5,15,1,8]$. We study the subset betting language in which a trader with a bet $i \in I$ pays $\$ b_{i}$ and bets on one of the two following types of scenarios: (1) one of the candidates ends up in a subset of positions $T$, or (2) one of the positions is occupied by a subset of candidates $S$. If the trader's bet is accepted, and the prediction is true, he gets $\$ 1$, and nothing if the prediction is false. Assuming that the surplus money of the traders go to the auctioneer, the revenue maximization problem for the auctioneer is to find a subset of bets that maximizes the revenue of the auctioneer in the worst possible outcome. This problem for subset betting has been posed as a question by Chen et. al [3]. We answer this question by proving that the revenue maximization problem is inapproximable within any factor. Next, we identify a special case of the problem, called singleton betting, that can be solved in polynomial time. In the singleton betting, each trader can bet $\$ b_{i}$ on the security that a candidate ends up in a position. We first give a necessary and sufficient condition for the existence of a solution with a positive profit in any possible outcome. Furthermore, we present a polynomial-time algorithm for finding the optimal solution of this problem. We first characterize an LP whose optimal integer solution is equal to our optimal solution in the betting problem. Then, we prove that we can change any optimal fractional solution of our LP to an integer solution with the same objective function in polynomial time. Finally, we consider the revenue maximization problem in settings where the auctioneer has some extra information about the set of possible outcomes of the market. We show how to use this extra information to find the optimal solution to maximize revenue. It is important to study this extension, since in realistic settings, the auctioneer may have some prior knowledge about the possible outcomes, and he/she should be able to use this information to find a better set of bets to accept. This observation is crucial in some realistic scenarios as it can increase the revenue of the auctioneer by a large amount.

In the probabilistic setting, we assume that the auctioneer has a probability distribution over the possible outcomes. In this case, instead of finding a subset of bets that guarantees some revenue, the auctioneer can try to guarantee a revenue of $x$ with high probability. This means that we are willing to take some risk and choose a set of bets that brings us revenue with high probability. This setting follows the idea of stochastic optimization $[13,10,23]$ in which we have a probability distribution over the possible scenarios, and need to find strategies that optimize in expectation, or with high probability. There are two types of objective functions in these settings. In the first type, the goal is to find a subset of bets that maximizes the expected revenue, given a probability distribution over the possible outcomes. In the second case, the goal is to maximize the probability of achieving at a revenue of at least $x$ (for a given parameter $x$ ). We first observe that the problem of maximizing the expected revenue in this model can be solved easily in polynomial time. However, we will show that maximizing the probability of achieving a pre-specified revenue $x$ is \#P-Complete.

\subsection{Related Work}

Permutation markets have been introduced by Chen, Fortnow, Nikolova, and Pennock [3]. They study two betting languages for this problem: the pair betting and the subset betting language. In a pair betting language, a trader with bet $i \in I$ pays $\$ b_{i}$ on pairs $(a, b)$ of candidates. If candidate $a$ ends up before candidate $b$ in the outcome, the trader gets 1 , and otherwise he gets nothing. The authors consider two types of problems: divisible, and indivisible. In indivisible problems, the auctioneer can accept or reject each bet. In divisible problems, the auctioneer can accept the bet to an extent $y_{i}$ where $y_{i}$ is a real number between 0 to 1. In the divisible setting, the authors show that the problem of maximizing revenue is polynomial-time solvable for subset betting, and is NP-complete for pair betting. They also consider the indivisible betting problem, and pose the approximability of the subset betting problem as an open question. After we wrote the first draft of this writeup, it was brought to our attention that Conitzer independently found an inapproximability result for this problem [4]. However, his proof is different from ours, and will appear in the journal version of the the paper by Chen et. al [3].

Prior to permutation markets, boolean-style markets have been studied by Fortnow et. al [6]. In these markets, a possible outcome of the market is one of the $2^{n}$ possible $0-1$ assignments to a set of $n$ variables. Each trader is allowed to bet on an arbitrary subset of these variables. Traders describe their bets in boolean formulas. The authors show that the matching problem in this setting is $c o-N P$-Complete for the divisible variant and $\sum_{2}^{P}$-Complete for the indivisible variant.

Another related work to betting markets is the market scoring rule mechanism defined in [11]. In this setting, a 
joint probability distribution across all outcomes is given, and traders bet on a combinatorial number of outcomes. One main difference between this setting and the framework considered in this paper is that the traders arrive sequentially, and the market maker pays to the last trader. In this setting, he may incur some loss. This is similar to our probabilistic setting in which the auctioneer may also incur loss with some probability. However, in the risk-free setting, when the trader accepts some bets, he/she does not bear any risk.

The risk-free setting considered in this paper is related to robust combinatorial optimization $[15,5,8]$ in which given a set of possible scenarios that can happen in the future, the goal is to find a strategy that optimizes the objective function in the worst scenario. A challenging and interesting aspect of permutation betting markets is that the number of possible outcomes is $n$ ! which is exponential in the size of the input. This is similar to the robust optimization framework with exponential number of scenarios. It has been proved that such robust optimization problems with exponential number of scenarios are harder to approximate [8]. The probabilistic setting considered in this paper is similar to stochastic optimization $[13,10,23]$ in which given a probability distribution over the possible scenarios that can happen in future, the goal is to find a strategy that optimizes the expected objective function. However, the literatures on both robust and stochastic optimization consider combinatorial optimization problems like network design and covering problems, and not the permutation problems considered in this paper.

The problem of allocating items to bidders in combinatorial auctions to maximize the auctioneer's revenue is considered in $[2,16,14,21,7]$. However, in contrast to our settings, the risk and uncertainty concepts are not considered in most of these works.

\subsection{Organization}

This paper is organized as follows. First, in Section 2, we formally define permutation markets and the subset betting problem. In Section 3, we define the subset betting problem, and prove its inapproximability. In Section 4, we first formally define the singleton betting problem, and give an algorithm to verify if there exists a subset of bets with positive revenue. Then, we provide a linear programming-based polynomial-time algorithm to maximize the revenue for the singleton betting problem. At the end of Section 4, we show how to solve the revenue maximization problem in the presence of some extra information about the possible outcomes of market. Finally, in Section 5, we define the probabilistic setting and present a positive remark and a negative result for this setting.

\section{PRELIMINARIES}

In this section, we formally define permutation betting markets and the subset betting problem.

Permutation betting markets. Permutation betting markets are markets in which the set of possible outcomes of the market is the set of all possible permutations of $n$ candidates. For example, the candidates can be horses in a race, and the outcome is the ranking of horses in an increasing order. In such markets, traders can bet on various types of securities for a future event. The result of the future event determines the outcome of the market. For example, the event could be a horse competition. In permutation markets, each security is a property of the ranking outcome. The security is not known before the event, and its truth will be revealed after the future event. For example, a security is "horse $A$ ends up in position 3". The auctioneer receives a set of bets on various types of securities, and can accept or reject each bet. Each bet $i$ consists of a bet value $b$ and a security $\phi . \quad b$ is the amount of money the trader is willing to pay if his/her bet is accepted. If the bet is accepted by the auctioneer, the trader pays $\$ b$ before the event, and after the event, if the security $\phi$ happens, e.g., if horse $A$ ends up in position 3, then, the trader gets $\$ 1$. The revenue of the auctioneer is defined as follows. If the auctioneer accepts a bet of value $b$ on a security $\phi$, if $\phi$ happens, the auctioneer's revenue from this bet is $b-1$, and if $\phi$ does not happen, the revenue from this bet is $b$. The (total) revenue of the auctioneer is the sum of his/her revenue from all accepted bets. In the risk-free setting, the goal of the auctioneer is to find a subset of bets that guarantees a positive revenue for him/her in any possible outcome. For example, if one trader bets on the event "horse $A$ ends up in position 2 " for $\$ 0.7$ (i.e., the trader pays $\$ 0.7$ ahead of time and gets $\$ 1$ if the event happens), and another trader bets on the event "horse $B$ ends up in position $2 "$ with $\$ 0.7$, then the set of all two bets is a risk-free set of bets for the auctioneer, since by accepting the two bets, in any possible outcome, the auctioneer has to pay $\$ 1$ to at most one trader, and thus the revenue of the auctioneer is $2 \times 0.7-1=0.4$. Our goal is to find a subset of bets for the auctioneer to accept in order to maximize the revenue.

Subset Betting. A subset betting permutation market allows two types of bets. Traders can either bet on a subset of positions a candidate may end up with, or they can bet on a subset of candidates that will occupy a particular position. In an instance of the subset betting problem, we are given a set of bets, $I$. A bet $i \in I$ of the first type is a triple $\left(b_{i}, x_{i}, Y_{i}\right)$ where $b_{i}$ is the amount of money that the trader is willing to pay, $x_{i}$ is the candidate he is bidding on, and $Y_{i}$ is a subset of positions. The trader gets $\$ 0$ if candidate $x_{i}$ does not end up in a position in set $Y_{i}$, and gets $\$ 1$ when candidate $x_{i}$ stands at one of the positions in set $Y_{i}$. A bet $j \in I$ of the second type is a triple $\left(b_{j}, X_{j}, y_{j}\right)$ where $b_{j}$ is the amount of money that the trader is willing to pay, $X_{j}$ is the set of candidates he is bidding on, and $y_{j}$ is a position. The trader gets $\$ 0$ if none of the candidates in $X_{j}$ ends up in position $y_{j}$, and gets $\$ 1$ if one of the candidates in set $X_{j}$ stands at position $y_{j}$.

\section{HARDNESS OF SUBSET BETTING}

In this section, we show that it is NP-hard to approximate the optimal revenue for subset bettings within any factor.

We say that an algorithm for the revenue maximization problem is a $c$-approximation, if for any input market with optimal revenue $x$, this algorithm runs in polynomial time and returns a solution with revenue not less than $c x$. We prove that the problem of maximizing revenue in subset bettings is not approximable within any multiplicative factor $c$ even in the special case that all bets are of second type, and $y_{j}$ is also equal to 1 for all bets. We do so by proving that in some instances of the problem, we can not even decide whether or not the optimal answer has positive profit for the auctioneer in every possible outcome. This fact implies that this problem can not be approximated. To see this, assume that the problem admits a $c$-approximation algorithm. 
Therefore, using this algorithm, we can verify whether $x$ is zero or a positive number. It remains to prove that verifying whether or not the revenue is positive is NP-hard.

DEFINITION 1. In the big independent set problem, we are given a graph $G$, with $n$ vertices, with no isolated vertex ( $a$ vertex of degree zero), and a number $k>n / 2$. The goal is to output 'Yes' when the graph has an independent set of size $k$, and 'No' otherwise.

Lemma 2. The big independent set problem is NP-Complete.

Proof. We prove the lemma using the NP-Completeness of the independent set problem. In the independent set problem, given a graph $G$ and an arbitrary input $k$, the goal is to find an independent set of size $k$ in $G$. Without loss of generality, we assume that $G$ has no isolated vertex. Now, we give a reduction from the independent set problem to the big independent set problem. Let the number of vertices and edges in $G$ be $n$ and $e$, respectively. Let $x$ be the size of the biggest independent set in $G$. We add $n$ patterns of $P_{3}$ (a path with 3 vertices) to $G$. This new graph has $n+3 n=4 n$ vertices and $e+2 n$ edges. Obviously, the size of the biggest independent set in this new graph is $2 n+x$ which is greater than $4 n / 2$. So the problem of finding the size of the biggest independent set in this new graph reduces to the big independent set problem. Thus, if there is a polynomial-time algorithm which solves the big independent set problem, we can find $2 n+x$ using this algorithm, and consequently $x$ can be found. As a result, the big independent set problem is NP-Complete.

Now, we can prove the main theorem of this section.

TheOREM 3. For any $c>0$, there is no c-approximation algorithm for subset betting problem with indivisible bets.

Proof. Let $(G, k)$ be an instance of the big independent set problem. We construct an instance of the subset betting problem as follows. For each edge between vertices $u$ and $v$ of $G$, we consider a candidate $c_{u, v}$ in our instance. Note that $c_{u, v}=c_{v, u}$. Let $\varepsilon$ be a positive number which satisfies inequalities $(k-1) \varepsilon<1<k \varepsilon$ and $n \varepsilon<2$. According to the fact that $k>n / 2$, we know that such an $\varepsilon$ exists. Now, for each vertex $v$ in $G$, we insert a trader in our instance of the subset betting problem. This trader's bet is a triple of form $\left(\varepsilon, X_{v}, 1\right)$ where $X_{v}=\left\{c_{v, u} \mid(v, u) \in E(G)\right\}$.

Now, we show that in order to have an output with a positive profit for any possible outcome, the auctioneer should not accept bets of two traders $u$ and $v$ which are adjacent in graph $G$. If the auctioneer accepts the bet of both traders $u$ and $v$, if a candidate $c_{u, v}$ stands at the first position (which is a possible outcome), he/she should pay $\$ 1$ to each of these two traders. Thus, in this case, he/she should pay $\$ 2$, but all the money that is given to the auctioneer is at most $n \times \varepsilon$ which is less than 2 . Note that there are $n$ traders, and each of them pays the auctioneer $\varepsilon$. This implies that if the auctioneer accepts the bets of two incident traders, there is a possible outcome in which his/her revenue is negative. This fact shows that the auctioneer should not accept bets of two adjacent traders $u$ and $v$. Therefore, the traders whose bets are accepted should form an independent set in $G$. There are possible outcomes in which we should pay one dollar. So, in order to have a positive revenue, we should accept at least $1 / \varepsilon$ number of bets. In other words, we should accept at least $k=\frac{1}{\varepsilon}$ bets which form an independent set of size at least $k$ in $G$. Therefore, this instance of the subset betting problem has a solution in which the auctioneer's revenue is always positive, the graph has an independent set of size $k$, and vice versa. Using the previous lemma, we know that this problem is NP-Complete. This fact proves that verifying if the revenue is positive or not cannot be done in polynomial time unless $P=N P$. Therefore, for any $c>0$, there is no $c$-approximation algorithm for the subset betting problem.

\section{THE SINGLETON BETTING PROBLEM}

In this section, we first formally define the singleton betting problem, and then give a linear-time algorithm for verifying if the auctioneer's revenue is positive. Next, we show that the problem of maximizing revenue for singleton betting can be solved via a linear programming formulation. Finally, we show that this polynomial-time algorithm can be used to solve the same revenue maximization problem in the presence of some extra information about the outcome of the market.

\subsection{Definitions and Notations}

The singleton betting problem. The singleton betting market problem is a special case of the subset betting problem in which players can bet on a singleton set of candidates for a single position. More formally, a singleton betting market allows traders to bet on a (single) position that a (single) candidate may end up with. Consider a set of candidates in a permutation market in which all $n$ ! permutations are possible outcomes, where $n$ is the number of candidates. In an instance of the singleton betting problem, we are given a set $I$ of bets that are submitted to the auctioneer by a set of traders. Each bet $i \in I$ is a triple $\left(b_{i}, x_{i}, y_{i}\right)$, where $x_{i}$ is a candidate, $y_{i}$ is a position, and $b_{i}$ is the amount which the trader $i$ is willing to pay for a unit share. Similar to the subset betting problem, if bet $i$ is accepted, trader $i$ pays $b_{i}$ before the outcome is revealed, and if candidate $x_{i}$ stands at position $y_{i}$ in the outcome, trader $i$ wins $\$ 1$ and wins $\$ 0$ otherwise. Given a set $I$ of bets, the auctioneer can accept or reject each of the bets. The goal of the auctioneer is to find a subset of bets that maximizes its revenue. To achieve this goal, we consider the following two problems: the existence problem, and the revenue maximization problem. In the existence problem, the auctioneer's goal is to find a subset of bets, called a risk-free subset, such that by accepting this subset, the auctioneer has a positive profit in any possible outcome. In the revenue maximization problem, the auctioneer's goal is to find a subset of bets such that accepting it, the auctioneer maximizes his/her minimum profit in every possible outcome. It is clear that the existence problem is a special case of the revenue maximization problem. There is a generalization in which any trader is allowed to order more than one share of security in her bet. In this case, the auctioneer is allowed to accept any subset of them. We can easily generalize our results to solve this problem.

In the following, we give a simple combinatorial algorithm for the existence problem, and an LP-based algorithm for the revenue maximization problem. First, we give some notation that will be used throughout this section.

Corresponding bipartite graph $G_{I}$. Given an instance of the singleton betting problem with a set of bets $I$, we construct a bipartite graph $G_{I}$. For every candidate, we 


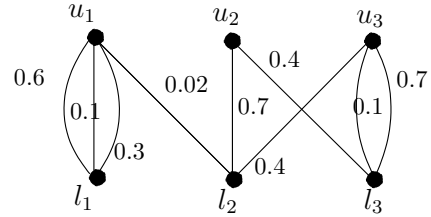

G
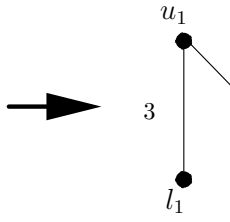

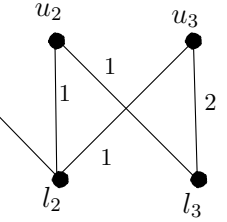

$G^{*}$
Figure 1: definition of $G^{*}$

place a vertex in the upper part of $G_{I}$ and for every position, we place a vertex in the lower part of $G_{I}$. Let $U^{G}$ denote the set of vertices in the upper part and $L^{G}$ denote the set of vertices in the lower part. We denote the $i$ th vertex of the upper part by $u_{i}$ and the $j$ th vertex of the lower part by $l_{j}$. Finally, for every triple $\left(b_{i}, x_{i}, y_{i}\right) \in I$, we put an edge between $u_{x_{i}} \in U^{G}$ and $l_{y_{i}} \in L^{G}$ with weight $b_{i}$. Note that it is possible to have multiple edges between two nodes in $G_{I}$.

Given a simple edge-weighted bipartite graph $G\left(U^{G}, L^{G}, E\right)$, let $w_{i, j}^{G}$ be the weight of the edge between vertices $u_{i} \in U$ and $l_{j} \in L$.

Given a multigraph $G(V, E)$, let $G^{*}\left(V^{*}, E^{*}\right)$ with edge weights $w^{*}$ be a simple edge-weighted graph with the same set of vertices, i.e, $V^{*}=V$ such that $w_{i j}^{*}$, the weight of the edge between vertices $i, j \in V^{*}$ in $G^{*}$, is equal to the number of edges between vertices $i$ and $j$ in $G$ (as shown in Figure 1).

Note that $w_{i, j}^{G^{*}}$ is equal to the number of edges between vertices $i$ and $j$ in $G$. Each bet has a corresponding edge in $G_{I}$. Therefore, when the auctioneer accepts a bet, we can also say that the auctioneer accepts the corresponding edge.

For example, consider the singleton betting market depicted in Figure 1. There are nine bets corresponding to edges of the bipartite graph. As a result, the set of bets, $I$ contains triples:

$(0.6,1,1),(0.1,1,1),(0.3,1,1),(0.02,1,2), \ldots,(0.1,3,3),(0.7,3,3)$

In this example, if the auctioneer accepts all bets, he/she gets $\$ 3.32$ before the outcome. If candidates 1,2 and 3 stand in positions 1,2 and 3 respectively, the auctioneers should pay $3+1+2=\$ 6$ to traders.

Accepted graph. If the auctioneer accepts a subset of bets, there is a subgraph in $G$ which is formed by the edges corresponding to the accepted bets. We call this graph the accepted graph. We say an auctioneer will win with respect to an accepted graph $H$, if accepting the bets corresponding to the edges of $H$ gives a positive revenue to the auctioneer in every possible outcome.

Graph theoretic preliminaries. In every graph $G$, let the value of the maximum weighted matching be $M_{G}$. Vector $\alpha=\left(\alpha_{1}, \alpha_{2}, \ldots, \alpha_{|V(G)|}\right)$ is called a weighted vertex cover of graph $G$ if for every edge $e=(i, j)$ we have $w_{e}^{G} \leq \alpha_{i}+\alpha_{j}$. The minimum weighted vertex cover is a weighted vertex cover which minimizes the sum $\sum_{i \in V(G)} \alpha_{i}$.

\subsection{The existence problem}

In this section, we give a necessary and sufficient condition for the existence problem which can be checked in linear time.
THEOREM 4. Given a set of bets I for the singleton betting problem, there exists a set of risk-free bets for the existence problem if and only if there exists a vertex $i \in V\left(G_{I}\right)$ and a set of edges $A$ such that (i) edges in $A$ are adjacent to vertex $i$, and each pair of edges in $A$ has only one endpoint in common which is $i$; and (ii) the total weight of edges in $A$ exceeds one, or equivalently $\sum_{e \in A} w_{e}^{G_{I}}>1$.

Proof. Proof of sufficiency: Suppose that there is a vertex $i$ with the desired properties. Without loss of generality, suppose $i$ is in the upper part of $G_{I}$. Assume that there exists a set $A$ of edges which are adjacent to $i$ and the sum of their weights is greater than 1 . If the auctioneer accepts the bets corresponding to edges in $A$ and rejects the other ones, the amount of money which is earned by him/her is greater than $\$ 1$. On the other hand, the auctioneer must pay at most $\$ 1$ in the worst case, because all the accepted bets have the candidate $i$ in common and their positions (the third element in the triples of bets) are distinct, so in each outcome the candidate $i$ stands at only one position, and we lose at most $\$ 1$.

Proof of necessity : Suppose there is no vertex $i$ with the desired property of the theorem and there is a subgraph $H$ of $G_{I}$ such that the auctioneer will win if he accepts the bets corresponding to edges of $H$. First, we find the subgraph $\hat{H}$ of $G_{I}$ such that the auctioneer will win if he accepts the corresponding bets of $\hat{H}$ and we have $w_{i, j}^{\hat{H}^{*}} \leq 1$, for all $i, j$. This means that there exists at most one edge between every pair of vertices $u_{i} \in U^{\hat{H}}$ and $l_{j} \in L^{\hat{H}}$ in $\hat{H}$. Finally, we prove that if a subgraph like $\hat{H}$ exists, we will reach a contradiction.

In order to prove the existence of $\hat{H}$, we need the following Lemma.

Lemma 5. Let $G$ be a weighted simple bipartite graph with integer weights. If the value of the maximum weighted matching in $G$ is $M_{G} \geq 1$, then there exists a vertex $i$ with the following property:

- If we decrease the weights of all edges adjacent to $i$ by 1 unit, the value of the maximum weighted matching in the remaining graph will be $M_{G}-1$.

Proof. The dual of the maximum weighted matching problem in a bipartite graph $G$ is the following problem: assign values $\alpha_{i}$ and $\beta_{j}$ to the vertices of $G$ ( $\alpha_{i}$ to vertex $u_{i} \in U$ and $\beta_{j}$ to vertex $\left.l_{j} \in L\right)$ such that for every edge $e=\left(u_{i}, l_{j}\right)$, we have that $\alpha_{i}+\beta_{j} \geq w_{i, j}$, and we also want to minimize the objective function $\sum_{u_{i} \in U} \alpha_{i}+\sum_{l_{j} \in L} \beta_{j}$. Based on the weak duality theorem, we know that the minimum feasible value of $\sum_{u_{i} \in U} \alpha_{i}+\sum_{l_{j} \in L} \beta_{j}$ is greater than or equal to $M_{G}$ in $G$. Consider the optimal dual solution $\alpha_{i}$ and $\beta_{j}$ for $u_{i} \in U$ and $l_{i} \in L$. Note that the weights of the edges in $G$ are integer, therefore, there exists an optimal solution in which all values of $\alpha_{i}$ and $\beta_{j}$ are integers. This is true, since the dual of the weighted matching is totally unimodular, and its integrality gap is 1 [22]. We also know that $M_{G} \geq 1$, so at least one $\alpha_{i}$ or one $\beta_{j}$ is greater than 0 . Without loss of generality, suppose $\alpha_{k}>0$, and because $\alpha_{k}$ is an integer number, we conclude that $\alpha_{k} \geq 1$. Now, we can decrease the weights of the edges adjacent to $u_{k}$ by 1 unit and let $G^{\prime}$ be the remaining graph. It is clear that $\left(\beta_{j}^{\prime}=\beta_{j}\right.$ for all $l_{j} \in L, \alpha_{i}^{\prime}=\alpha_{i}$ for all $u_{i} \in U, u_{i} \neq u_{k}$ and $\left.\alpha_{k}^{\prime}=\alpha_{k}-1\right)$ is a feasible solution for the dual problem 
in graph $G^{\prime}$ with value $\sum_{u_{i} \in U^{G}} \alpha_{i}^{\prime}+\sum_{l_{j} \in L^{G}} \beta_{j}^{\prime}=M_{G}-1$. Therefore, the value of every weighted matching in $G^{\prime}$ is not greater than $M_{G}-1$. On the other hand, consider the maximum weighted matching in $G$ with value $M_{G}$. It is clear that the value of this matching in $G^{\prime}$ is $M_{G}-1$. So the value of the maximum weighted matching in $G^{\prime}$ is $M_{G}-1$.

Now we return to the proof of Theorem 4. Consider a graph $H$ and assume that the auctioneer accepts the bets corresponding to the edges of $H$. (Note that we assume that the auctioneer will win by accepting these bets). Let the value of the maximum weighted matching in $H^{*}$ be $M_{H^{*}}$. It is clear that for some permutations, the auctioneer must pay $M_{H *}$ to the traders. On the other hand, the auctioneer gets $\sum_{e \in H} w_{e}^{H}$ amount of money from traders at first. Since the auctioneer is seeking a risk-free subset, we should have:

$$
M_{H^{*}}<\sum_{e \in H} w_{e}^{H}
$$

If $H^{*}$ has an edge with weight greater than 1 , there are at least two edges in $H$ between the endpoints of that edge with weight greater than one. We repeat the following procedure iteratively, until there is no edge with weight greater than 1 in $H^{*}$.

- We know that there exists a vertex $i$ in $H^{*}$ with the desired property of Lemma 5 . For every vertex $j$, remove one of the edges between vertices $i$ and $j$ in $H$. Let $\dot{H}$ be the remaining graph. According to Lemma 5 , if we decrease the weights of edges adjacent to $i$ in $H^{*}$ by 1 unit, the value of the maximum weighted matching in $H^{*}$ will decrease by exactly 1 unit. Therefore, we have $M_{\dot{H}^{*}}=M_{H *}-1$. We assume that there is no vertex with the desired property of Theorem 4. Therefore, the sum of the weights of the removed edges from $H$ is not greater than 1, and we have $\sum_{e \in \dot{H}} w_{e}^{\dot{H}} \geq \sum_{e \in H} w_{e}^{H}-1$. Using Equation 1 we conclude:

$$
M_{\dot{H}^{*}}=M_{H *}-1<\sum_{e \in H} w_{e}^{H}-1 \leq \sum_{e \in \dot{H}} w_{e}^{\dot{H}}
$$

This proves that if the auctioneer accepts the bets corresponding to the edges of $\dot{H}$, he wins. Therefore, we can replace $H$ by $\dot{H}$ and repeat this procedure iteratively until we reach a graph $H^{*}$ with no edge weight greater than one.

This proves that there exists a graph $H$ such that the auctioneer wins with respect to $H$, and there is at most one edge between any pair of vertices in $H$. Now, we construct a network flow $F$ using the graph $H$ as follows.

1. Add two vertices $s$ and $t$ to $H$. Let $s$ be the source of our network flow and $t$ be its sink.

2. Put an edge between $s$ and each vertex $u_{i}$ in the upper part of $H$ with capacity of 1 .

3. Put an edge between each vertex $l_{j}$ in the lower part of $H$ and $t$ with capacity of 1 .

4. Let the capacity of each edge from vertex $u_{i}$ in the upper part to vertex $l_{j}$ in the lower part be equal to $w_{u_{i}, l_{j}}^{H}$.

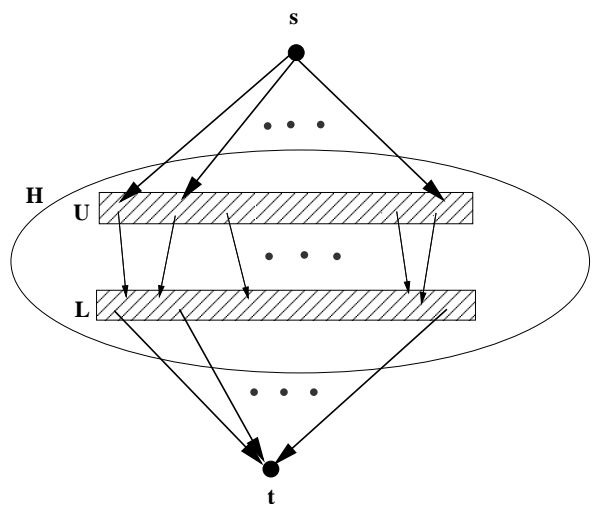

Figure 2: Constructing the network flow $F$ using graph $H$

We know that there is no vertex with the desired property of Theorem 4. Therefore, for every vertex $i$, the sum of the weights of the edges adjacent to $i$ in $H$ is not greater than 1. So, we have a flow with value $\sum_{e \in H} w_{e}^{H}$ in the network flow $F$. Construct a new network flow $F^{\prime}$ by rounding up the capacities of edges in $F$. It is clear that the value of the maximum flow in $F^{\prime}$ is not less than the value of the maximum flow in $F$, because we do not decrease the capacities. On the other hand, we can see that the value of the maximum flow in $F^{\prime}$ is equal to the value of the maximum weighted matching in $H^{*}\left(M_{H^{*}}\right)$. Knowing these facts, we can conclude:

$$
M_{H^{*}}=\max \text { flow in } F^{\prime} \geq \max \text { flow in } F \geq \sum_{e \in H} w_{e}^{H}
$$

which is a contradiction (see Equation 1.)

Verifying the necessary and sufficient condition of Theorem 4 for all vertices in graph $G_{I}$ can be done in running time $O(|I|+m+n)$ where $n$ and $m$ are the number of candidates and positions respectively. As a result, Theorem 4 gives a linear-time algorithm for the existence problem.

Now we are ready to solve the generalization in which traders can order more than one share of security. In the existence problem, we only need to compute the sum of weights of edges incident to a specific vertex $u$. Note that If we submit $C$ copies of a bet, these are $C$ parallel edges in $G_{I}$ with 2 common vertices. Therefore we should consider only one of them in our calculations. Therefore this generalization is not computationally harder.

\subsection{The revenue maximization problem}

In this section, we propose a polynomial-time algorithm for finding a subset of bets with the maximum guaranteed revenue to the auctioneer. The algorithm is based on a linear programming (LP) formulation. We first characterize an LP whose optimal integer solution is equal to our optimal solution in the singleton betting problem. We relax the linear program to a fractional linear program, and then prove that we can change any optimal fractional solution of our LP to an integer solution with the same objective function in polynomial-time. Note that in the revenue maximization problem, we have a weighted bipartite graph $G$ with multiple edges each of which corresponds to one bet, and we want 

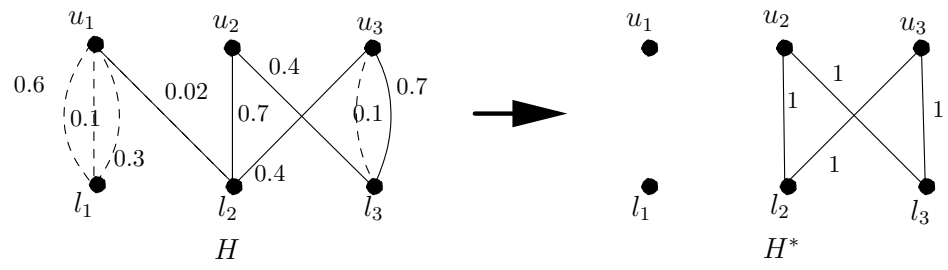

Figure 3: A solution with revenue 0.2

to find a subgraph $H$ of $G$ that maximizes:

$$
\sum_{e \in H} w_{e}^{H}-M_{H^{*}}
$$

In other words, if the auctioneer accepts the bets of the edges of graph $H$, he earns $\sum_{e \in H} w_{e}^{H}$ amount of money from traders, and he should pay $M_{H^{*}}$ in the worst case outcome. For example, consider the singleton betting market shown in Figure 1. If the auctioneer accepts bets $(0.7,2,2),(0.4,2,3)$, $(0.4,3,2)$ and $(0.7,3,3)$, the accepted graph is $H$ that is shown in Figure 3. Note that $\sum_{e \in H} w_{e}^{H}$ is equal to 2.2, and the maximum weighted matching in graph $H^{*}, M_{H^{*}}$ is 2 . So the revenue is $2.2-2=0.2$ in this case.

Now we characterize the structure of the maximum revenue solution. Let the subgraph $H \subseteq G$ be the maximum revenue solution. Consider the minimum weighted vertex cover of $H^{* 1}$. Assume that the value $\alpha_{i}$ is assigned to the vertex $u_{i} \in U^{H^{*}}$, and $\beta_{j}$ is assigned to the vertex $l_{j} \in L^{H^{*}}$ in this weighted vertex cover.

LEMma 6. In the optimal accepted graph $H, w_{i, j}^{H^{*}}$ is equal to $\min \left(w_{i, j}^{G^{*}}, \alpha_{i}+\beta_{j}\right)$ for every pair of vertices $i$ and $j$.

Proof. We know that $H$ is a subgraph of $G$, thus $w_{i, j}^{H^{*}}$ is not greater than $w_{i, j}^{G^{*}}$. On the other hand, the values $\alpha_{1}, \alpha_{2}, \ldots, \alpha_{|V(H)|}$ and $\beta_{1}, \beta_{2}, \ldots, \beta_{|V(H)|}$ form a weighted vertex cover of graph $H^{*}$, so we have that $w_{i, j}^{H^{*}} \leq \alpha_{i}+\beta_{j}$.

For the sake of contradiction, assume $w_{i, j}^{H^{*}}$ is not equal to $\min \left(w_{i, j}^{G^{*}}, \alpha_{i}+\beta_{j}\right)$ for some $i$ and $j$, thus $w_{i, j}^{H^{*}}<w_{i, j}^{G^{*}}$ and $w_{i, j}^{H^{*}}<\alpha_{i}+\beta_{j}$. The value $w_{i, j}^{H^{*}}$ is integer, for any pair of vertices $i$ and $j$. Therefore, by definition, $\alpha_{i}$ and $\beta_{j}$ are also integers, for every $i$ and $j$. Thus, $w_{i, j}^{H^{*}}+1 \leq \alpha_{i}+\beta_{j}$. We can add one of the edges between $i$ and $j$ in $G-H$ to $H$. Note that such an edge exists because we assume that $w_{i, j}^{H^{*}}<w_{i, j}^{G^{*}}$. It implies that the value of $w_{i, j}^{H^{*}}$ increases by one. But, it is clear that the minimum weighted vertex cover of $H^{*}$ is still a weighted vertex cover. Using the fact that the value of the maximum weighted matching is equal to the value of the minimum weighted vertex cover, we conclude that the value of $\sum_{e \in H} w_{e}^{H}-M_{H^{*}}$ will increase by adding one of edges between vertices $i$ and $j$ to the optimal accepted graph $H$. This contradicts the optimality of $H$.

Lemma 6 implies that using values of $\alpha_{i}$ and $\beta_{j}$, we can determine the value $w_{i, j}^{H^{*}}$ by setting it to $\min \left(w_{i, j}^{G^{*}}, \alpha_{i}+\beta_{j}\right)$. Now, we can write an integer linear program whose optimal solution determines our optimal solution for the singleton betting problem. In this ILP, we want to find the values of $\alpha_{i}$ and $\beta_{j}$. We also want to choose edges which should be added to the optimal subgraph $H$. For ease of notation, let

\footnotetext{
${ }^{1}$ See the definition of $G^{*}$ in the preliminaries.
}

$w_{i, j, t}^{G}$ be the weight of the $t^{t h}$ edge between vertices $i$ and $j$ in $G$. Without loss of generality assume that the edges between vertices $i$ and $j$ are sorted in decreasing order with respect to their weight such that we have $w_{i, j, t}^{G^{*}} \geq w_{i, j, t+1}^{G^{*}}$. The following program is the ILP which helps us in computing the minimum weighted vertex cover:

$$
\begin{aligned}
\max & \left(\sum w_{i, j, t}^{G} y_{i, j, t}-\sum x_{i}-\sum x_{j}^{\prime}\right) \\
& \sum_{t=1}^{w_{i, j}^{G}} y_{i, j, t}=Y_{i, j} \quad \forall i \in U, j \in L \\
& Y_{i, j} \leq x_{i}+x_{j}^{\prime} \quad \forall i \in U, j \in L \\
& x_{i}, x_{j}^{\prime} \geq 0 \quad \forall i \in U, j \in L \\
& y_{i, j, t} \in\{0,1\} \quad \forall i \in U, j \in L, 1 \leq t \leq k_{i j}
\end{aligned}
$$

The ILP variables $x_{i}, x_{j}^{\prime}, y_{i, j, t}$ and $Y_{i, j}$ are defined as follows:

- $x_{i}$ is the value of $\alpha_{i}$ in the minimum weighted vertex cover of $H^{*}$.

- $x_{j}^{\prime}$ is the value of $\beta_{j}$ in the minimum weighted vertex cover of $H^{*}$.

- $Y_{i, j}$ is the value of $w_{i, j}^{H^{*}}$ which is equal to number of edges between vertices $i$ and $j$ in $H$.

- $y_{i, j, t}$ is a number which is equal to 0 or 1 , and indicates whether the $t^{t h}$ edge between $i$ and $j$ in $G$ belongs to $H$.

By strong duality, the value of the maximum weighted matching is equal to the value of the minimum weighted vertex cover. Since the ILP variables $x$ and $x^{\prime}$ correspond to the vectors of the minimum weighted vertex cover $(\alpha$ and $\beta$ ), the value $\sum x_{i}+\sum x_{j}^{\prime}$ in the objective function of the ILP is equal to $M_{H^{*}}$. As a result, the optimal solution of the integer linear program 5 characterizes the maximum revenue of the singleton betting problem. In order to solve the integer linear program 5, we relax the integer constraints $y_{i, j, t} \in\{0,1\}$ to linear fractional constraints $0 \leq y_{i, j, t} \leq 1$. As a result, we get the following linear programming relaxation:

$$
\begin{aligned}
\max & \left(\sum w_{i, j, t}^{G} y_{i, j, t}-\sum x_{i}-\sum x_{j}^{\prime}\right) \\
& \sum_{t=1}^{w_{i, j}^{G^{*}}} y_{i, j, t}=Y_{i, j} \quad \forall i \in U, j \in L \\
& Y_{i, j} \leq x_{i}+x_{j}^{\prime} \quad \forall i \in U, j \in L \\
& x_{i}, x_{j}^{\prime} \geq 0 \quad \forall i \in U, j \in L \\
& 0 \leq y_{i, j, t} \leq 1 \quad \forall i \in U, j \in L, 1 \leq t \leq k_{i j}
\end{aligned}
$$

We can solve the linear program 6 . Now, the question is how to round the solution of 6 and construct an integer solution for program 5 . The following Lemma 7 shows that the integrality gap of this linear program is 1 and any solution of this LP can be rounded to an integer solution in polynomial time without changing the value of the objective function. Here, we prove this fact by showing that LP 6 is totally unimodular.

LEMMA 7. The integrality gap of LP 6 is 1 and an optimal integer solution of ILP 5 can be found in polynomial-time by solving the LP relaxation 6 . 
Proof. Here, we prove this lemma by showing that the LP is totally unimodular.

There are four types of variables in LP 6, i.e. $y_{i, j, t}, Y_{i, j}, x_{i}, x_{j}^{\prime}$. Let $v$ be a vector that contains all types of variables. We can write the constraints of LP 6 as a matrix inequality $A v \leq b$ where $A$ and $b$ are defined as follows: $A$ is a matrix whose number of rows and columns are equal to the number of constraints and variables in the LP respectively, and entries of $A$ correspond to coefficients in the linear constraints of this LP. The vector $b$ contains the right hand side values of the constraints. There are some equality constraints in LP 6 . We can use some slack variables, and replace these equalities with some inequalities, so the constariants can be written in the inequality form $A v \leq b$. By the way, these slack variables do not disturb the totally unimodularity property of this inequality system. It is well known that in order to prove that the integrality gap of LP 6 is 1, it suffices to show that $A$ is totally unimodular $[18,24]$. This fact also implies a polynomial-time algorithm for rounding any fractional solution of LP 6 to an optimal integer solution to ILP 5. For contradiction, assume $A$ is not totally unimodular. In that case, $A$ should have a square submatrix with determinant not equal to 0,1 or -1 . Suppose $K$ is the smallest square submatrix of $A$ with this property.

It is not hard to see that each row or column of $K$ has at least two non-zero entries. Since, if there is a row (or column) with only one non-zero entry $a$ ( $a$ is either 1 or -1 ), we can say that the absolute value of determinant of $K$ is equal to the absolute value of determinant of $K^{\prime}$ where $K^{\prime}$ is the matrix that is obtained from $K$ by removing the row and column of entry $a$. So the determinant of $K^{\prime}$ is also not equal to 0,1 or -1 . This contradicts with the assumption that $K$ is the smallest square submatrix with determinant not equal to 0,1 or -1 . According to the fact that each row of $K$ has at least two non-zero entries, we conclude that rows corresponding to constraints like $y_{i, j, t} \leq 1$ or $y_{i, j, t} \geq 0$ are not selected as rows of $K$. Therefore, without loss of generality, we can delete these rows from $A$, and assume that $K$ is a submatrix of the remaining matrix. Since each column of $K$ also has at least two non-zero entries, we can say that the columns corresponding to variables $y_{i, j, t}$ are not selected as columns of $K$, because each of these columns in the remaining matrix contains exactly one non-zero entry. Similarly we can remove these columns, and assume that $K$ is a submatrix of the remaining matrix. Now consider a row corresponding to a constraint of the form $\sum y_{i, j, t}=Y_{i, j}$. Since the columns of variables of the form $y_{i, j, t}$ are removed later, the row corresponding to this constraint has only one non-zero entry. Again we can remove these rows from our matrix. In the remaining matrix, the columns of variables of form $Y_{i, j}$ has only one non-zero entry, therefore we remove these columns too. The remaining matrix has only rows of constraints of form $Y_{i, j} \leq x_{i}+x_{j}^{\prime}$ and columns of variables of form $x_{i}$ or $x_{j}^{\prime}$. Note that each row of this matrix has exactly two non-zero variable with the same sign. Partition the columns into two sets $B=\left\{x_{1}, x_{2}, \ldots, x_{n}\right\}$ and $C=$ $\left\{x_{1}^{\prime}, x_{2}^{\prime}, \ldots, x_{n}^{\prime}\right\}$. One of those two non-zero entries belongs to a column in set $B$, and the other one belongs to a column in set $C$. According to [12], the determinant of any square submatrix of this matrix, including $K$ is equal to 0 , 1 or -1 which is again a contradiction. Therefore we conclude $A$ is totally unimodular, and thus, the integrality gap of the corresponding integer linear program is equal to 1 .
Using ILP 5 and the result of Lemma 7, it follows that the revenue maximization problem for singleton betting is polynomial-time solvable. We conclude this section by the following theorem.

THEOREM 8. The revenue maximization problem for the auctioneer in singleton betting can be solved in polynomial time.

Note that linear program 6 is a small polynomial-size linear program that can be solved very efficiently in practice as well. This is different from the exponential-size linear programming formulation of Chen et.al [3] for divisible variant of the subset betting.

Now consider the case that traders can order more than one share of security. The only change we should make is that the constraints of the form $y_{i, j, t} \in\{0,1\}$ should be replaces with $y_{i, j, t} \in\{0,1, \ldots, C\}$ in ILP5 where $C$ is the number of copies of a bet that the corresponding trader orders. It is clear that the integrality gap of LP6 remains 1 in this case.

\subsection{The revenue maximization problem with extra information}

In this section, we study the revenue maximization problem for singleton betting when we are given a set of pieces of extra information about the outcome of the betting market. Each piece of the extra information is a forbidden pair $(x, y)$ which means that candidate $x$ never ends up in position $y$. The auctioneer may gain this type of information from various sources, or can predict such forbidden pairs with such a high confidence that he/she does not bear any risk by assuming these forbidden pairs. Let $F$ be the set of these pairs. Given a set of forbidden pairs, a possible outcome we can illustrated by a perfect matching among candidates and positions in which no forbidden pair appears. In that case, the auctioneer can use this information in his/her favor and in order to solve the revenue maximization problem, he should take into account such extra information. In this section, we show that the LP-based revenue maximization algorithm from the previous section can be extended to solve the revenue maximization problem with extra information.

Before stating the algorithm, we discuss an example. Consider the singleton betting market in Figure 1. Suppose we know that candidates 1 and 2 do not stand at position 1 in any possible outcome (see Figure 4 ). In other words, the edges $(1,1)$ and $(2,1)$ are forbidden pairs. Using this extra information, we can say that the candidate 3 necessarily stands at position 1 . Therefor, the auctioneer gains the maximum revenue by accepting all bets except bet $(0.02,1,2)$. The auctioneer gets $\$ 3.3$ before the outcome, and will pay at most $\$ 1$ to the traders after the outcome.

First, we show how to calculate the minimum revenue over all possible outcomes with respect to a given accepted graph $H$. Then we propose a linear programming method to find, an accepted graph which maximizes this minimum revenue over the possible outcomes. Note that a possible outcome can be shown by a perfect matching $M$ among candidates and positions that does not use the forbidden pairs. The sum of weights of edges that are in both $M$ and $H^{*}$ (See definition of $G^{*}$ in Subsection 4.1) is the value that we should pay to the traders in this outcome. Therefore, in order to find the minimum revenue over all possible outcomes, we should find the maximum weighted perfect matching in $H^{*}$ 

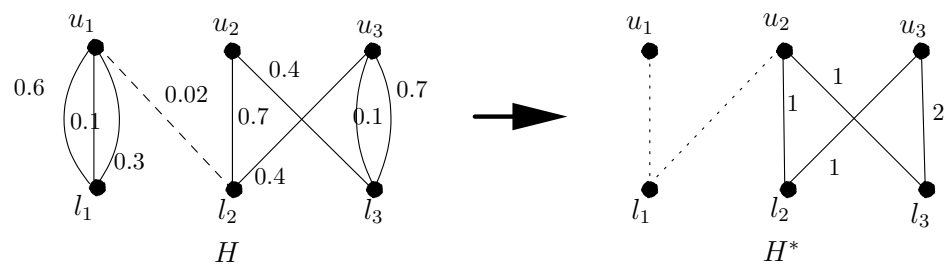

Figure 4: Best solution with extra information

without using forbidden edges. We can solve this by finding the integer solution of the following LP:

$$
\begin{aligned}
\max & \left(\sum w_{i, j}^{H^{*}} x_{i, j}\right) \\
& \sum_{j=1}^{n} x_{i, j}=1 \quad \forall i \in U \\
& \sum_{i=1}^{n} x_{i, j}=1 \quad \forall j \in L \\
& x_{i, j}=0 \quad \forall(i, j) \in F \\
& x_{i, j} \geq 0 \quad \forall i \in U, j \in L
\end{aligned}
$$

We consider the dual of the above program:

$$
\begin{aligned}
\min & \left(\sum \alpha_{i}+\sum \beta_{j}\right) \\
& \alpha_{i}+\beta_{j} \geq w_{i, j}^{H^{*}} \quad \forall(i, j) \notin F \\
& \alpha_{i}+\beta_{j}+\delta_{i, j} \geq w_{i, j}^{H^{*}} \quad \forall(i, j) \in F
\end{aligned}
$$

In the above LP, $\alpha_{i}$ is the variable corresponding to the constraint of candidate $i \in U, \beta_{j}$ is the variable corresponding to the constraint of position $j \in L$, and $\delta_{i, j}$ is the variable corresponding to the constraint of the forbidden edge $(i, j)$. Note that all variables including $\delta_{i, j}$ can get arbitrarily large positive or negative values. Variables $\delta_{i, j}$ does not contribute in the cost function, so by setting $\delta_{i, j}=+\infty$, we can eliminate the constraints of the form $\alpha_{i}+\beta_{j}+\delta_{i, j} \geq w_{i, j}^{H^{*}}$. Therefore, we can rewrite the dual program as follows:

$$
\begin{aligned}
\min & \left(\sum \alpha_{i}+\sum \beta_{j}\right) \\
& \alpha_{i}+\beta_{j} \geq w_{i, j}^{H^{*}} \quad \forall(i, j) \notin F
\end{aligned}
$$

This program finds the values $\alpha_{i}$ and $\beta_{j}$ such that for each non-forbidden edge $(i, j)$, we have $\alpha_{i}+\beta_{j} \geq w_{i, j}^{H^{*}}$. Since LP 7 is the similar to the LP of the maximum weighted matching problem, the integrality gap of LP 7 is 1 [24]. Therefore the best fractional solution of the dual program 9 is equal to the maximum integer solution of LP 7 .

Now we propose an algorithm to find an accepted graph maximizing this minimum revenue over the possible outcomes. With the same argument as Lemma 6, we can use values of $\alpha_{i}$ and $\beta_{j}$ of dual program 9 to determine the value $w_{i, j}^{H^{*}}$ by setting it to $\min \left(w_{i, j}^{G^{*}}, \alpha_{i}+\beta_{j}\right)$. Now, we can write an integer linear program whose optimal solution determines our optimal solution for the singleton betting problem with extra information. The ILP is very similar to ILP 5. Again we want to find the values of $\alpha_{i}$ and $\beta_{j}$ and we also want to choose edges which should be added to the optimal subgraph $H$. The following program is the ILP for computing the minimum weighted vertex cover in this setting:

$$
\begin{aligned}
\max & \left(\sum w_{i, j, t}^{G} y_{i, j, t}-\sum x_{i}-\sum x_{j}^{\prime}\right) \\
& \sum_{t=1}^{w_{i, j}^{G^{*}}} y_{i, j, t}=Y_{i, j} \quad \forall i \in U, j \in L \\
& Y_{i, j} \leq x_{i}+x_{j}^{\prime} \quad \forall(i, j) \notin F \\
& y_{i, j, t} \in\{0,1\} \quad \forall i \in U, j \in L, 1 \leq t \leq k_{i j}
\end{aligned}
$$

THEOREM 9. The revenue maximization problem for the auctioneer in singleton betting with extra information can be solved in polynomial time.

Proof. Similar to the proof of Lemma 7, we can show that the constraint matrix of ILP 10 is totally unimodular. Therefore if we relax the integer constraints $y_{i, j, t} \in\{0,1\}$ to linear fractional constraints $0 \leq y_{i, j, t} \leq 1$, we can solve this linear programming relaxation and round it to optimal integer solution of ILP 10. The optimal solution of ILP 10, corresponds to the maximum revenue of singleton betting problem with extra information.

\section{THE PROBABILISTIC SETTING}

In this section, we study the betting problem in the probabilistic setting. We first define the problem formally. Assume that the auctioneer has a probability distribution $q$ over the possible outcome permutations, i.e.,

$\sum_{\sigma}$ is a permutation $q(\sigma)=1$. Given a probability distribution $q$, a desired revenue $x$, and a desired probability $0 \leq p \leq 1$, we consider the following two problems:

DEFINITION 10. In the max-expected subset betting problem, given a probability distribution $q$ and a set $I$ of subset bets, our goal is to find a subset $S$ of bets $I$ such that accepting bets in $S$ maximizes the expected revenue of the auctioneer.

DEFINITION 11. In the max-probability singleton betting problem, given a probability distribution $q$ over the possible outcomes, a desired revenue $x$, a desired probability $0 \leq p \leq$ 1 , and a set I of simple bets, our goal is to accept a subset $S$ of bets in $I$ in order to have revenue $x$ with probability at least $p$, and refuse to return a subset if there does not exist such a subset.

Here, we observe that max-expected subset betting problem can be solved easily, but the max-probability singleton betting problem is \#P-complete.

In order to solve the max-expected subset betting problem, for any subset $S \subseteq I$, let $E(S)$ be the expected revenue when the auctioneer accepts the bets in set $S$. For every bet $i \in I$, let $E(i)$ be the revenue when we accept only bet $i$. Based on the linearity of the expectation, $E(S)=$ $E\left(\cup_{i \in S} i\right)=\sum_{i \in S} E(i)$. Thus, in order to maximize $E(S)$, we should add a bet $i \in I$ into $S$ iff $E(i)>0$. Therefore, it suffices to compute $E(i)$ for each bet $i$. Let $p_{i}$ be the probability that the security of bet $i$ happens. Given the probability distribution $q$, we can estimate $p_{i}$ by sampling the probability distribution $q$. Thus, we can estimate $E(i)=b_{i}-p_{i}$. Note that in the probabilistic model, the input can have exponential size, but we can assume that the probabilities are given in a oracle model. Next, we prove the hardness of the max-probability singleton betting problem. 
THEOREM 12. The max-probability singleton betting problem is \#P-Complete.

Proof. We reduce the problem of counting the number of perfect matchings in a bipartite graph to the maxprobability singleton betting problem. In fact, we consider a simpler version of this problem which is equivalent to the original one. Suppose we are given a bipartite graph $G$ and a number $k$ and we are asked whether there are at least $k$ perfect matchings in $G$. We construct an instance of the max-probability singleton betting problem as follows. Suppose each edge in $G$ is between sets $X$ and $Y$ where $|X|=|Y|=n$. For each vertex in $x_{i} \in X$, consider a candidate $a_{i}$ in our instance. For each edge $\left(x_{i}, y_{j}\right)$ in $G$, we put a bet in our instance of the form $\left(2, a_{i}, j\right)$ which means that this trader is willing to pay $\$ 2$ for this bet, and the trader wins $\$ 1$ if the candidate $a_{i}$ stands in position $j$. In this instance, we set $x=2 E-n+1$ where $E$ is the number of edges in $G$. We also define $p$ to be $1-\frac{k-1}{n !}$. It is obvious that in the optimum solution we should accept all the bets. By definition, we are asked if the revenue is at least $2 E-n+1$ with probability $1-\frac{k-1}{n !}$. Equivalently, we are asked if there are at most $k$ matchings of size $n$ in $G$. Thus max-probability singleton betting problem can solve the problem of calculating the number of perfect matchings in a bipartite graph in polynomial time which is a \#P-Complete problem.

\section{CONCLUSION}

In this paper, we studied the subset and singleton betting problems for permutation markets in the risk-free and probabilistic settings. We also considered the singleton betting problem with extra information in which the auctioneer has some certain knowledge of the possible outcome of the market. We showed that maximizing revenue for the subset betting problem is not approximable, but the singleton betting problem is solvable by solving a linear programming relaxation and rounding its solution, even in the presence of certain knowledge about the outcome of the market. An interesting question is to study revenue maximization problem with extra knowledge about the set of outcomes for different betting languages like pair betting and subset betting. It is also interesting to consider other types of extra information about the possible outcomes. This extra information may include some probability distribution on certain properties of the outcome.

Acknowledgments. The third author thank Evdokia Nikolova for introducing permutation betting markets.

\section{REFERENCES}

[1] J. E. Berg, R. Forsythe, F. D. Nelson, and T. A. Rietz. Results from a dozen years of election futures markets research. In C. A. Plott and V. Smith, editors, Handbook of Experimental Economic Results, 2001.

[2] P. Cramton, Y. Shoham and R. Steinberg. Combinatorial Auctions. MIT Press, Cambridge, MA, 2005.

[3] Y. Chen, L. Fortnow, E. Nikolova, D. Pennock. Betting on Permutations, Proceedings of the ACM Conference on Electronic Commerce, 2007.

[4] D. Pennock. Personal Communications.

[5] K. Dhamdhere, V. Goyal, R. Ravi, and M. Singh. How to pay, come what may: Approximation algorithms for demand-robust covering problems. FOCS, 2005.
[6] R. Forsythe, T. A. Rietz, and T. W. Ross. Wishes, expectations, and actions: A survey on price formation in election stock markets. Journal of Economic Behavior and Organization, 39:83-110, 1999

[7] U. Feige. On maximizing welfare when utility functions are subadditive, STOC, 2006.

[8] U. Feige, K. Jain, M. Mahdian, and V. S. Mirrokni. Robust Combinatorial Optimization with Exponential Number of Scenarios. Integer Programming and Combinatorial Optimization, 2007.

[9] L. Fortnow, J. Kilian, D. Pennock, M. P. Wellman. Betting boolean-style: A framework for trading in securities based on logical formulas. Decision Support Systems, 39(1):87-104, 2004.

[10] A. Gupta, M. Pal, R. Ravi, and A. Sinha. Boosted sampling: Approximation algorithms for stochastic optimization. In STOC, pages 170-178, 2004.

[11] R.D. Hanson. Combinatorial information market design. Information Systems Frontiers, 5(1):107-119, 2003.

[12] I. Heller, C.B. Tompkins. An extension of a theorem of Dantzig's, in: H.W. Kuhn, A.W. Tucker (Eds.), Linear Inequalities and Related Systems. Princeton University Press, Princeton, NJ, 1956, pp. 247-254.

[13] N. Immorlica, D. Karger, M. Minkoff, and V. S. Mirrokni. On the costs and benefits of procrastination: Approximation algorithms for stochastic combinatorial optimization problems. In SODA, 2004.

[14] B. Lehmann, D. Lehmann, and N. Nisan. Combinatorial Auctions with Decreasing Marginal Utilities, ACM EC 2001.

[15] Y. Nikulin. Robustness in combinatorial optimization and scheduling theory: An annotated bibliography. Technical Report SOR-91-13, Statistics and Operation Research, 2004.

[16] N. Nisan, T. Roughgarden, E. Tardos and V. Vazirani. Algorithmic Game Theory. Cambridge University Press, 2007.

[17] D. M. Pennock, S. Lawrence, C. L. Giles, and F. A. Nielsen. The real power of artiŕcial markets. Science, 291:987-988, February 2002.

[18] C. Papadimitriou, and K. Steiglitz. Combinatorial Optimization: Algorithms and Complexity N.Y. : Dover Publications, Inc., 1998.

[19] C. Plott and S. Sunder. Efficiency of experimental security markets with insider information: An application of rational expectations models. Journal of Political Economy, 90:663-98, 1982

[20] C. Plott and S. Sunder. Rational expectations and the aggregation of diverse information in laboratory security markets. Econometrica, 56:1085-1118, 1988.

[21] T. Sandholm. Algorithm for optimal winner determination in combinatorial auctions. Artificial Intelligence, 135:1-54, 2002.

[22] A. Schrijver. Total Dual Integrality of Matching Forest Constraints. Combinatorica, 20:575-588, 2000.

[23] D. Shmoys and S. Swamy. Stochastic optimization is (almost) as easy as deterministic optimization. In FOCS, 2004.

[24] A. Schrijver, Combinatorial Optimization - Polyhedra and Efficiency, Springer-Verlag, Berlin, 2003. 